\title{
Persistent and transient cost efficiency-an application to the Swiss hydropower sector
}

\author{
Massimo Filippini $^{1,2} \cdot$ Thomas Geissmann $(10){ }^{1,3} \cdot$ William H. Greene ${ }^{4}$
}

Published online: 27 November 2017

(c) The Author(s) 2017. This article is an open access publication

\begin{abstract}
Electricity prices on the European market have decreased significantly over the past few years, resulting in a deterioration of Swiss hydropower firms' competitiveness and profitability. One option to improve the sector's competitiveness is to increase cost efficiency. The goal of this study is to quantify the level of persistent and transient cost efficiency of individual firms by applying the generalized true random effects (GTRE) model introduced by Colombi et al. (Journal of Productivity Analysis 42(2): 123-136, 2014) and Filippini and Greene (Journal of Productivity Analysis 45(2): 187-196, 2016). Applying this newly developed GTRE model to a total cost function, the level of cost efficiency of 65 Swiss hydropower firms is analyzed for the period between 2000 and 2013. A true random effects specification is estimated as a benchmark for the transient level of cost efficiency. The results show the presence of both transient as well as persistent cost inefficiencies. The GTREM predicts the aggregate level of cost inefficiency to amount to $21.8 \%$ (8.0\% transient, $13.8 \%$ persistent) on average between 2000 and 2013. These two components differ in interpretation and implication. From
\end{abstract}

Thomas Geissmann

tgeissmann@ethz.ch

1 Department of Management, Technology and Economics, Centre for Energy Policy and Economics, ETH Zurich, Zurich, Switzerland

2 Department of Economics, University of Lugano, Lugano, Switzerland

3 MIT Energy Initiative, Joint Program on the Science and Policy of Global Change, Massachusetts Institute of Technology, Cambridge, MA, USA

4 Department of Economics, Stern School of Business, New York University, New York, NY, USA an individual firm's perspective, the two types of cost inefficiencies might require a firm's management to respond with different improvement strategies. The existing level of persistent inefficiency could prevent the hydropower firms from adjusting their production processes to new market environments. From a regulatory point of view, the results of this study could be used in the scope and determination of the amount of financial support given to struggling firms.

Keywords Efficiency measurement $\cdot$ Stochastic frontier analysis $\cdot$ Persistent and transient cost efficiency $\cdot$ Hydropower

JEL classification $\mathrm{C} 01 \cdot \mathrm{C} 23 \cdot \mathrm{D} 23 \cdot \mathrm{L} 94 \cdot \mathrm{Q} 25$

\section{Introduction}

Ever since Switzerland's electrification at the beginning of the 20th century, hydropower has been the country's main domestic source of electricity. Over time, Swiss hydropower firms have consolidated their position as reliable, cost effective and renewable base and peak load electricity producers. Hydropower also has enabled Switzerland to play an active role on the European electricity market. The pursued business models can roughly be summarized as follows: run-of-river plants produce base load electricity while storage and pump-storage plants use their natural water inflows to help covering electricity demand at peak hours, usually occurring at noon and early evening. All three technology types not only produce for the domestic market, but also are extensively involved in exporting activities to the European grid. A special role is accorded to 
the pump-storage plants, whose business model exploits the spread between peak and off-peak electricity prices. In addition of using natural water inflows for electricity generation, they pump water into their reservoirs during off-peak hours at favorable prices-often during nighttime -by consuming electricity directly from the high voltage grid. This electricity is partly sourced from the European electricity market, and especially from the French nuclear fleet. At peak load times, the water is turbinated again and the generated electricity is sold at comparatively high prices.

This business model was very successful until 2008 . Then, the economic crisis, the low price of coal, the low price of $\mathrm{CO}_{2}$ certificates not reflecting the emission's external costs and the subsidy system for renewable energies such as wind and photovoltaics have led to a significant drop in overall market prices for electricity. In this context, the competitiveness of the coal power plants, which do not have to cover all of their external costs, has increased significantly. In addition, the spread between peak and off-peak electricity prices on the European electricity markets have decreased or at some hours even completely disappeared. In this context, the competitiveness of the coal power plants has increased significantly. Furthermore, since 2009 the Swiss electricity market has been partially liberalized, giving electricity distribution companies and large customers consuming more than 100 MWh per year the possibility to purchase electricity from a producer of their choice in Switzerland or other European countries or to buy electricity directly on the European spot markets. Of course, this reform has increased the level of competition among the Swiss hydropower firms resulting in a pressure to reduce production costs. In January 2015, the decoupling of the Swiss Franc from the Euro has led to an additional reduction in margins, since the electricity traded on a European level is denominated in Euros. For these reasons, a growing share of hydropower plants has started to incur financial losses in recent years. In the current competitive context, it is of immediate importance for them to identify strategies to increase competitiveness by reducing production costs.

One possibility to achieve such goal is to improve the level of cost efficiency, which, as discussed in Colombi et al. (2011, 2014), can be split into two parts: a persistent and a transient one. The persistent part captures cost inefficiencies which do not vary with time. These could be inefficiencies due to recurring identical management mistakes, structural problems within the electricity generation process or factor misallocations that are difficult to change over time. On the other hand, the transient component represents cost inefficiencies varying with time, e.g., singular, non-systematic management mistakes. In the short- to medium-run, a firm's leverage is expected to be mainly on the improvement of the transient part of cost efficiency.

Information on the level of cost efficiency is of importance not only for the firms, but also for the Swiss federal government. In fact-because of the distortions ${ }^{1}$ on the European electricity market-the Swiss parliament decided in 2015, under some circumstances, to financially support hydropower firms in financial distress. However, the political process of specifying the details of such a subsidization system is still ongoing. From an economic policy point of view, it is important to grant such subsidies only to firms operating already with a high degree of efficiency. Hence, knowledge on the level of cost efficiency supports the government in avoiding subsidizing inefficient hydropower firms.

Despite the fact that hydropower still is the world's dominant source of renewable energy, the scientific literature only comprises a few published studies on the productive efficiency of hydropower firms. ${ }^{2}$ Banfi and Filippini (2010) study the cost structure and level of cost efficiency of an unbalanced panel of 43 Swiss hydropower firms observed from 1995 to 2002. Using a translog variable cost function, they employ the true random effects model proposed by Greene (2005a, b), i.e., a stochastic frontier approach. The explanatory variables considered are: total amount of electricity produced, number of plants per firm, price of labor and capital stock. Furthermore, four binary indicators are added to the model controlling for different types of technology. ${ }^{3}$ Their empirical results indicate economies of utilization as well as the presence of cost inefficiency. By also using a variable cost function approach, Barros and Peypoch (2007) examine the cost efficiency of a balanced panel of 25 Portuguese hydropower plants, all of them belonging to the main Portuguese utility, for the years 1994 to $2004 .{ }^{4}$ From the econometric point of view, these authors also use a translog functional form and

\footnotetext{
1 The distortions, as discussed previously, are mainly due to the subsidization of new renewables and the presence of non-covered external costs.

${ }^{2}$ For a publication summarizing several studies on efficiency measurement in the general electricity generation sector see, e.g., Barros (2008). More recent contributions to the measurement of efficiency in the electricity generation sector were made, e.g., by Yang and Pollitt (2009) (China - coal plants-DEA), Sueyoshi et al. (2010) (USAcoal plants-DEA), Liu et al. (2010) (Taiwan-thermal plantsDEA), Shrivastava et al. (2012) (India-coal plants-DEA), See and Coelli (2012) (Malaysia-thermal plants-SFA) and Chen et al. (2015) (China-thermal plants-Bayesian SFA).

${ }^{3}$ The cost function specified in Banfi and Filippini (2010) was also used by Filippini and Luchsinger (2007) to quantify the economies of scale of the Swiss hydropower sector using cost share equations and the seemingly unrelated regression concept of Zellner (1962).

${ }^{4}$ Using the same data and looking at the years 2001 to 2004, Barros (2008) analyzes and decomposes the productivity of the hydropower firm by using data envelopment analysis (DEA) applied to a production function.
} 
the true random effects model. Finally, Barros et al. (2013) analyze the level of cost efficiency of a relatively small panel of twelve Chinese hydropower firms for the period 2000 to 2010 using a total cost function in translog functional form. They use a stochastic frontier latent class model to take into account possible differences in the unobserved production technology affecting costs. The estimation results obtained indicate the presence of three distinct groups of firms. Their choice to use a latent class model is an interesting approach for the case where the firms' production technology is not directly observed.

Most of the empirical literature so far has fallen short of a differentiation of the persistent and transient component of productive efficiency. Also the aforementioned studies provide only empirical information on the transient, but not the persistent, part of cost efficiency. With an empirical orientation, this paper's main goal therefore is to measure the level of persistent and transient cost efficiency for a sample of Swiss hydropower firms by estimating a homothetic translog frontier total cost function. We use a new and representative panel of Swiss hydropower firms. In a firm's context, the persistent part of productive inefficiency may be due a variety of factors like regulations, investments in inefficient machines or infrastructure or lasting habits of the management to waste inputs. The transient part of inefficiency on the other hand, for example, may stem from temporal behavioral aspects of the management or from a non-optimal use of some machines. Such distinction and measurement of the two components of overall cost efficiency is interesting because it allows the firms to elicit their cost saving potential in the short- as well as the long-run. Furthermore, from a policy point of view, it can be of interest when reforming the electric power sector.

The simultaneous measurement of the level of persistent and transient cost efficiency is based on a four component stochastic frontier (SF) model. This model has been introduced by Colombi et al. $(2011,2014)$ and Kumbhakar et al. (2014). Filippini and Greene (2016) proposed an innovative way of estimating the four component SF model by using maximum simulated likelihood. The contribution of the present paper to the scientific literature is threefold and mainly empirically oriented. Firstly, from an econometric perspective, we provide a stand-alone empirical application of the novel estimation method proposed by Filippini and Greene (2016). Secondly, a rich cost model specification is used, explicitly controlling, e.g., for the technological heterogeneity between run-of-river, storage and pump-storage plants. Thirdly, firm-level information on the two categories of persistent and transient cost inefficiency can help the government to design an effective subsidy policy by granting financial aids only if the firms meet predefined efficiency standards in both categories.
The structure of this paper is as follows: Section 2 contains a description and gives and overview of the data used for the empirical analysis. Section 3 describes the empirical cost model as well as the chosen functional form, and Section 4 presents the econometric estimation methodologies. Results are summarized in Section 5. Finally, Section 6 concludes and discusses the findings.

\section{Data}

Hydropower electricity generation in Switzerland is mainly based on approximately 600 plants operated by several dozen hydropower firms, ${ }^{5}$ contributing roughly 55 to $60 \%$ to the total domestic electricity generation. Most of these plants (ca. 80\%) are of run-of-river type, with storage and pump storage plants making up the remaining share (BFE 2013). The Swiss hydropower firms are organized according to a specific structure, with the largest part of them being so-called partner firms ("Partnerwerke" in German). ${ }^{6}$ These partner firms sell the generated electricity to Swiss utilities who in turn are mainly active in the distribution, sales and trading of electricity in Switzerland as well as on the European electricity market.

The econometric analysis is based on an unbalanced ${ }^{7}$ panel data set comprising 65 hydropower firms over the time period of 2000 to 2013. Most of these firms are "Partnerwerke". The financial data was extracted from the yearly annual reports of these firms and extended by firm specific technical information contained in the "Statistik der Wasserkraftanlagen der Schweiz" (WASTA), which is published annually by the Swiss Federal Office of Energy (BFE 2013). By means of this technical information, hydropower firms are classified into three distinctive categories to account for heterogeneities in the production processes of the power plants. The three categories, representing the dominating power plant type operated by a firm, are: run-of-river, storage and pump storage. Following Filippini et al. (2001), the classification is conducted as follows: Storage power firm produce at least $50 \%$ of their expected electricity generation by storage power plants, whereby the share of the pump capacity is smaller or equal

\footnotetext{
5 A hydropower firm may have several plants under operation. A plant represents a building containing one or more turbines. Geographically, these plants usually are located in a close perimeter to each other.

${ }^{6}$ A partner firm has two main characteristics. First, it has been created by several electricity companies (mother firms) with the only purpose to produce electricity. Second, the generated electricity is sold to a fixed, cost-covering price (including a fair return on investment) to the mother firms, depending on the ownership share.

7 The underlying reasons for the data to be unbalanced are, for example, firm mergers or annual reports not being obtainable anymore due to, e.g., ownership changes. None of the sample attrition was due to firms ceasing production.
} 

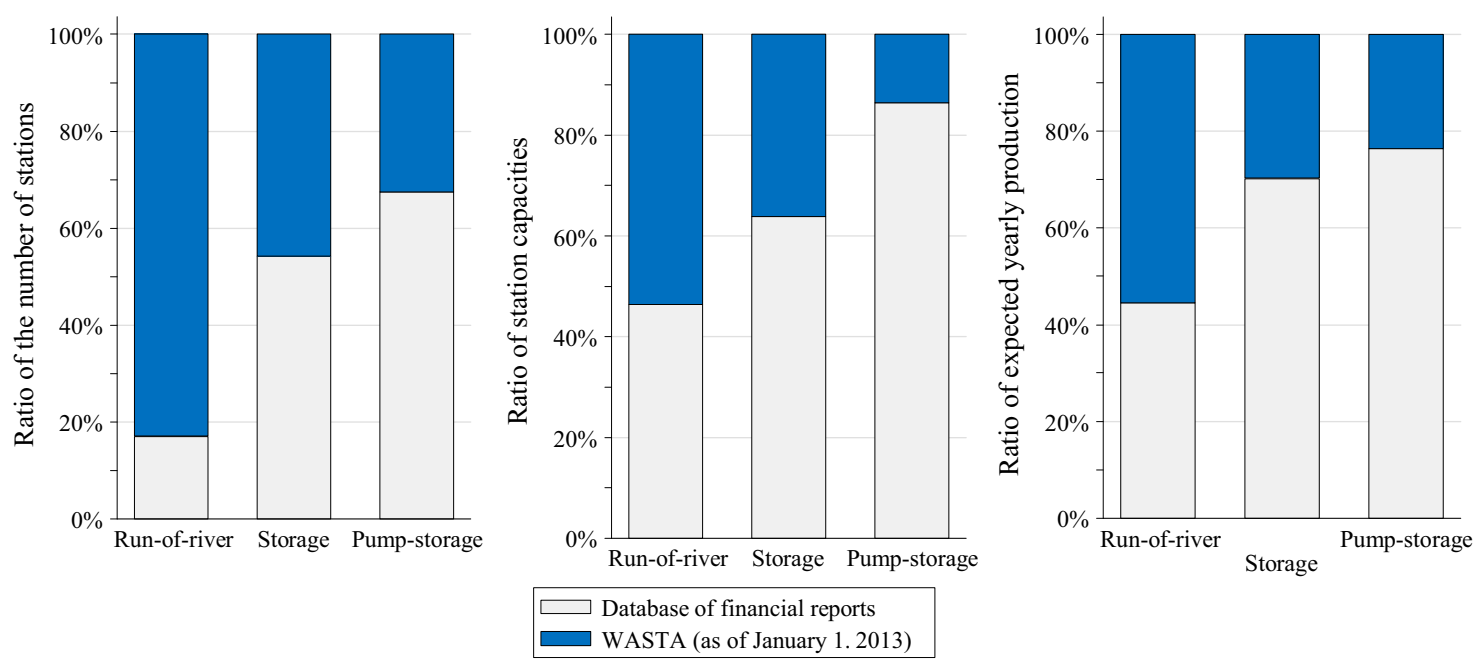

Fig. 1 Representativeness of the sample in terms of the number of stations, the turbine capacity and the expected generation in 2013. Note: Fig. 1 shows the degree, to which firms of the sample are representative of the population of Swiss hydropower stations with a turbine capacity of at least $300 \mathrm{~kW}$. This population of stations is contained in the WASTA. For example, the right bar of the right panel indicates our sample to represent roughly $80 \%$ of the expected yearly generation of the population of pump-storage plants to $10 \%$ of the total maximum possible generator capacity. A pump storage power firm produces at least $50 \%$ of its expected electricity generation by storage power plants, whereby the share of the pump capacity is larger than $10 \%$ of the total maximum possible generator capacity. All other firms are considered to be of type run-of-river.

A specific firm type does not imply all plants operated by this firm being of same kind; it rather indicates the dominating plant type. The plant types of the firms classified to be of type run-of-river are relatively homogenous, i.e., most of these firms exclusively or to a large extent operate run-ofriver plants. Furthermore, this firm type runs comparatively few plants, usually one or two. This is in contrast to the plants run by the storage and pump storage firms, which are more diverse in type and larger in number per firm. The average share of run-of-river type firms in our sample is $58 \%$. The share of storage type firms is 19.9 and $22.1 \%$ for pump storage type firms. Our sample of hydropower firms represents the Swiss hydropower sector quite well, especially in terms of the turbine capacity and expected generation (cf. Fig. 1). For the period 2000 to 2013, we observe approximately $60 \%$ of the total expected generation of the Swiss hydropower plants with a turbine capacity larger than $300 \mathrm{~kW}$.

The power plants usually are not older than 50 years or have undergone at least once a major remodeling during the last five decades. The highest share of plants in our sample is located in Alpine cantons, which corresponds to the general distribution of hydropower plants in Switzerland. For topological and hydrological reasons the storage and pump-storage firms are mainly situated in the Alpine cantons.

\section{Empirical specification}

\subsection{Parametrization of the cost function}

The frontier total cost function represents the minimum cost a firm potentially could achieve in producing a given amount of output by using a given technology and facing given input prices. Usually, none or only a few firms are operating at the cost frontier. Failure to do so implies the existence of cost inefficiency. ${ }^{8}$ In what follows, a stochastic frontier total cost function is estimated using panel data. Such estimation of the frontier necessitates the specification of a parametric model, the choice of a functional form and finally, the identification of an econometric approach.

The cost of a firm operating one or more hydropower plants is influenced by several factors such as output, factor prices, size of the reservoir, production technology (storage, pump-storage or run-of-river), age or the number of hydropower plants in a firm's portfolio. Therefore, the cost function for the Swiss hydropower firms may be specified as

$$
C=c\left(Y, P_{L}, P_{W}, P_{K}, P_{E}, F, N, D_{S}, D_{P}, t\right),
$$

where $C$ are the total generation costs. Firm $i$ and time $t$ subscripts are dropped for notational simplicity. The single output, $Y$, is gross electricity generation in $\mathrm{kWh}$. The price

\footnotetext{
$\overline{8}$ In this study we estimate a cost frontier function which does not allow distinguishing between technical and allocative inefficiency. See, e.g., Kumbhakar and Lovell (2000), Coelli et al. (2005) or Fried et al. (2008) for an in depth description of the concept of technical and allocative efficiency as well as overall cost efficiency.
} 
of labor is represented by $P_{L}$, the price of water by $P_{W}$ and the residual price of capital by $P_{K}$. The price of energy used in electricity production is $P_{E}$. To capture additional heterogeneities in the production process, the cost function includes on the one hand the firm's average load factor $F$. This variable helps to differentiate between, e.g., a run-of-river or storage firm, as the latter usually shows a much lower load factor than the former. ${ }^{9}$ To further control for the presence of different types of hydropower firms, technology fixed effects $D_{S}$ and $D_{P}$ are included into the model. These indicate whether a firm uses predominantly storage $\left(D_{S}\right)$ or pump-storage $\left(D_{P}\right)$ plants for electricity generation, with run-of-river representing the reference firm type. ${ }^{10}$ With run-of-river firms bunching up in the Swiss midlands, and storage and pump storage firms being concentrated in Alpine regions, these variables in addition capture heterogeneity in terms of the production environment. Finally, the number of plants under operation, $N$, measures the impact on cost of jointly operating several plants. Even though electricity generation by hydropower is based on mature technologies, a time trend $t$ is included to capture exogenous technological change. Total costs are based on an accounting approach. Hence, it is worth noting that the framing of the cost function follows a firm oriented perspective rather than a society oriented one, i.e., the cost function does not account for possible external costs arising from the electricity generation process.

Under the assumption of cost minimizing firms, a cost function should satisfy the properties of concavity and linear homogeneity in input prices. Furthermore, it should be non-decreasing in output and input prices. Linear homogeneity in input prices can be imposed by

\footnotetext{
9 The yearly load factor considered in the model is inherently connected to a power plant's technology. We are aware that the load factor could be considered to be endogenous. However, the owners of an hydropower plant built as partner firm have to share the yearly generated electricity according to long-term agreements. One particularity of a partner firm is that-at the time of construction-the partners agree to buy a predefined share of the yearly generated electricity over a plant's complete lifecycle, and independent of demand. Further, the amount of water in the reservoir and the quantity of water flowing in the river are exogenously given by meteorological and geographic conditions for most of the technologies. Therefore, the amount of generated electricity $(Y)$ and subsequently the yearly load factor can be considered to be exogenous.

${ }^{10}$ Another approach to capture heterogeneities in the production process would consist of an application of a latent class model, as done in, e.g., Barros et al. (2013). However, we decided against this approach, because we observe technological heterogeneity. We are also more interested in the distinction between persistent and transient inefficiency. We believe that the latent class model is not completely appropriate for the estimation of a cost function based on a small sample and that our cost model specification and econometric approach sufficiently controls for heterogeneities in the production processes.
}

normalizing cost and input prices by one of the input prices. The other properties are to be verified once the translog cost function has been estimated. We justify the necessary assumption of output levels being exogenous to hold based on the monopolistic structure of the electricity market. Firms faced public service obligations for most of the years considered in the empirical analysis, i.e., the general production plan was defined on an annual basis, instead of a daily basis depending on market conditions.

We decided to use a translog functional form (Berndt and Christensen 1973; Christensen et al. 1973) to estimate the cost function in Eq. (1). In a preliminary analysis, we tried to estimate a fully flexible version of the translog functional form. However, due to the presence of highly correlated variables in the cost model, such as output, load factor or number of stations, such model specification suffered from multicollinearity. For this reason, we decided to estimate a homothetic version of the translog cost function, a version that is more parsimonious in the number of coefficients to be estimated. Based on Eq. (1) the homothetic version of the translog cost function can be expressed as shown in Eq. (2).

$$
\begin{aligned}
c= & \alpha+\beta_{Y} \ln Y+\sum_{X=\{L, W, K\}} \beta_{X} \ln P_{X}+\beta_{F} F+\beta_{N} \ln N \\
& +\frac{1}{2}\left(\beta_{Y Y}(\ln Y)^{2}+\sum_{X=\{L, W, K\}} \beta_{X X}\left(\ln P_{X}\right)^{2}+\beta_{F F} F^{2}+\beta_{N N}(\ln N)^{2}\right) \\
& +\sum_{X=\{W, K\}} \beta_{L X} \ln P_{L} \cdot \ln P_{X}+\beta_{W K} \ln P_{W} \cdot \ln P_{K}+\beta_{Y F} \ln Y \cdot F \\
& +\beta_{Y N} \ln Y \cdot \ln N+\beta_{F N} F \cdot \ln N+\beta_{D S} D_{S}+\beta_{D P} D_{P}+\beta_{t} t+u+v .
\end{aligned}
$$

For notational simplicity, the unit index $i$ as well as the time index $t$ are omitted, and $\alpha$ is the intercept. Linear homogeneity in prices is imposed by normalizing total costs and factor price variables by the price of energy. Because of its comparative robustness with regard to outliers, the variables' median value was chosen as point of approximation (i.e., variables are divided by their respective median value). Hence, the estimated coefficients represent elasticities at the sample's respective median values. As will be explained in Section 4, the concept of the stochastic frontier analysis splits the error term $\varepsilon$ into an inefficiency component $u$ and the usual white noise term $v$, i.e., $\varepsilon=u+\nu$.

\subsection{Variable definitions}

Total generation costs include water fees, amortization, financial expenses, profit before taxes, material and external services, personnel costs, costs for energy and grid 
access, other taxes and dues as well as other costs. All financial variables have been deflated to real 2010 values using the Swiss producer price index published by BFS (2014). The price of labor, $P_{L}$, is defined as personnel costs divided by the number of employees. For firms with missing information on the price of labor, a year and region specific price proxy is constructed, thereby allowing for structural differences in salaries between geographic regions. ${ }^{11}$ The price of water, $P_{W}$, is defined as the ratio of the sum of water fees and other concession fees to a firm's total turbine capacity. Following (Friedlaender and Wang Chiang 1983), the capital price, $P_{K}$, is estimated as residual costs divided by the turbine capacity, which serves as a proxy for the capital stock. Residual costs are defined as total costs minus labour costs, energy costs and water costs, i.e., they include material and external service costs, allowances for depreciation, financial expenses and profits before taxes. ${ }^{12}$ Finally, a single energy price, $P_{E}$, is assumed for all hydropower firms. In fact, energy costs are mainly composed of expenditures on electricity. The presence of a uniform European electricity market justifies the assumption of firms facing a cross-section wise constant price of electricity.

Some firms activated additional capital allowances on non-depreciable investments before the opening of the electricity market to increase the level of competitiveness, especially around the beginning of the new millennium. As some of these additional allowances exceed usually observed numbers by a multiple, they cause a significant distortion of the respective firms' cost structure. To avoid the distorting effect of such special accounting measures, extraordinary allowances in one year were corrected for by adjusting the amortization rate of that year to the firm specific average amortization rate of the other years. ${ }^{13}$ Furthermore, if mother companies delivered pump energy free of charge, these opportunity costs were valued and

\footnotetext{
11 This labor price proxy represents the year specific median labor price of all firms of a region with observed labor prices. The seven geographic regions of Switzerland are defined as follows: Lake Geneva region (1), midland (2), Northwestern Switzerland (3), Zurich (4), Eastern Switzerland (5), Central Switzerland (6), Ticino (7). Furthermore, for the firms located on the German and French border, two separate regions ( 8 and 9 ) are defined.

12 Profits before taxes are assumed to represent the equity yield rate. Unfortunately, we do not have all the information necessary to estimate a capital price based on the economic approach of opportunity costs of capital. Hence, the adopted definition of the proxy of the price of capital based on the available accounting data is rather broad.

13 Such amortization cost correction affected 8 firms in a total of 14 periods, i.e., ca. $1.7 \%$ of the observations. The amortization rate is the ratio of the amortization costs to the sum of the reported book value of fixed assets (excluding assets under construction) and realized investments. We chose the book value because not all hydropower firms publish numbers on asset acquisitions. However, the use of the book value implies a non-linear depreciation schedule, while hydropower firms usually depreciate linearly.
}

subsequently added to total costs. ${ }^{14}$ Finally, the load factor $F$ is formed by a division of $Y$, the gross electricity generation, by the total turbine capacity, whereby the latter is multiplied by the number of hours per year. The variables' descriptive statistics are given in Table 1.

\section{Estimation methodologies}

In what follows, the level of cost efficiency of a sample of Swiss hydropower firms is estimated using a parametric approach, i.e., the stochastic frontier analysis (SFA). ${ }^{15}$ Econometric SFA models for panel data allow both the estimation of the transient and persistent part of the cost inefficiency. Moreover, parametric approaches are suitable in cases of unobserved heterogeneity influencing production processes, like environmental characteristics. ${ }^{16}$

The measurement of inefficiency using SFA has a longstanding tradition in the literature. The SFA methodology dates back to the end of the 1970s when first contributions -at that time focusing exclusively on cross-sectional data —were made by Aigner et al. (1977), Meeusen and Broeck (1977) and Battese and Corra (1977). Since then, the concept of SFA was extended significantly to the longitudinal setting by Pitt and Lee (1981), Cornwell et al. (1990) and Greene (2005a, b). ${ }^{17}$ Recently, Colombi et al. $(2011,2014)$ and Kumbhakar et al. (2014) have proposed a new stochastic frontier model that simultaneously distinguishes between two parts of productive efficiency, i.e., a persistent and a transient part.

\footnotetext{
$\overline{14}$ This correction only affects five firms in a total of 39 periods, i.e. ca. $4.5 \%$ of the observations. The correction for non-allocated pump energy charges at a rate of 3 cents per kWh accounts for the fact that consumed pump energy is of different quality than the electricity generated by a pump storage plant: From 2000 to 2013 (our sample period), water usually was pumped at nighttime when electricity prices were low. Electricity generation, however, focused on peak load times, usually at noon and in the evening, since these periods were characterized by high prices.

15 The literature on the measurement of a firm's productive efficiency roughly can be divided into two main methodological strands: the parametric and the non-parametric analysis. SFA represents the prevalent parametric approach, whereas the data envelopment analysis (DEA) constitutes the most prominent non-parametric approach. Nonparametric approaches do not necessitate an a priori specification of a functional form and use linear programming, while parametric approaches are based on econometric concepts, allowing them to differentiate between unobserved heterogeneity and inefficiency. Furthermore, non-parametric approaches are not able to distinguish in a satisfactory way between technical and allocative cost inefficiency, which together form the overall cost inefficiency.

16 A more extensive discussion on methodological differences as well as an extensive description of SFA models can be found in, e.g., Greene (2008), Coelli et al. (2005) or Kumbhakar and Lovell (2000). ${ }^{17}$ See Filippini and Greene (2016) for a review of several stochastic frontier models for panel data.
} 
Table 1 Descriptive statistics of the variables

\begin{tabular}{lllll}
\hline & Mean & Std. dev. & Min. & Max. \\
\hline Total costs $C$ [million CHF] & 24.20 & 30.96 & 0.32 & 195.92 \\
Electricity generation $Y$ [GWh] & 433.38 & 484.06 & 5.82 & 2695.00 \\
Price of labor $P_{L}[\mathrm{kCHF}$ per employee] & 127.80 & 19.10 & 74.90 & 247.15 \\
Price of water $P_{W}[\mathrm{CHF}$ per kW] & 45.41 & 34.64 & 0.54 & 336.98 \\
Price of capital $P_{K}[\mathrm{CHF}$ per kW] & 145.90 & 108.22 & 17.00 & 739.68 \\
Load factor $F$ [index] & 0.492 & 0.331 & 0.104 & 2.608 \\
Number of stations $N$ & 2.49 & 2.03 & 1 & 13 \\
Time trend $t$ & 7.46 & 4.02 & 1 & 14 \\
Storage fixed effect $D_{S}$ & 0.199 & 0.400 & 0 & 1 \\
Pump storage fixed effect $D_{P}$ & 0.221 & 0.415 & 0 & 1
\end{tabular}

Note: This table presents descriptive statistics of the variables of the cost function given in Eq. (1). CHF indicates Swiss Francs. The statistics are based on the full sample of observations. Monetary values are given in real 2010 values
In this paper, we decided to use two alternative stochastic frontier models for panel data. The first is the true random effects model (TREM hereafter) proposed by Greene $(2005 \mathrm{a}, \mathrm{b})$ that produces values of the productive inefficiency that vary over time (transient inefficiency). The TREM includes group-specific random effects to capture any time-invariant unobserved heterogeneity. Further, as in the basic stochastic frontier model proposed by Aigner et al. (1977), the error term is composed of two parts: a stochastic error capturing the effect of noise and a one-sided nonnegative disturbance representing the level of inefficiency. The TREM has the advantage to control for time-constant unobserved heterogeneity. On the other side, any timeinvariant component of inefficiency is absorbed in the group-specific random effects. Therefore, the TREM tends to produce an estimate of the level of transient inefficiency.

The second econometric model is the generalized true random effects model (GTREM). To estimate this four way random component model, at least four approaches can be identified. For instance Colombi et al. (2014) describe an estimation method based on full maximum likelihood. Kumbhakar et al. (2014) present a three-step method of moments estimator. Tsionas and Kumbhakar (2014) propose an estimation procedure based on Bayesian Markov chain Monte Carlo methods. Filippini and Greene (2016) present a maximum simulated likelihood technique. ${ }^{18}$ In their approach, the log likelihood function noted in Colombi et al. (2014) is simplified by exploiting the formulation of Butler and Moffitt (1982) in the simulation, where the loglikelihood function is computed using Hermite quadrature. The log-likelihood function then is estimated by maximum simulated likelihood using Halton sequences. Instead of using four unique disturbance terms as in Colombi et al.

\footnotetext{
$\overline{18}$ Recently, Badunenko and Kumbhakar (2017) further extended the four component model to accommodate determinants of inefficiency.
}

Table 2 Distributional assumptions of the stochastic cost frontier models

\begin{tabular}{|c|c|c|}
\hline & TREM & GTREM \\
\hline Full random error $\varepsilon_{i t}$ & $\left\{\begin{aligned} \varepsilon_{i t} & =r_{i}+u_{i t}+v_{i t} \\
u_{i t} & \sim N^{+}\left(0, \sigma_{u}^{2}\right) \\
v_{i t} & \sim N\left(0, \sigma_{v}^{2}\right) \\
r_{i} & \sim N\left(0, \sigma_{r}^{2}\right)\end{aligned}\right.$ & $\begin{aligned} \varepsilon_{i t} & =r_{i}+h_{i}+u_{i t}+v_{i t} \\
u_{i t} & \sim N^{+}\left(0, \sigma_{u}^{2}\right) \\
v_{i t} & \sim N\left(0, \sigma_{v}^{2}\right) \\
r_{i} & \sim N\left(0, \sigma_{r}^{2}\right) \\
h_{i} & \sim N^{+}\left(0, \sigma_{h}^{2}\right)\end{aligned}$ \\
\hline Persistent inefficiency estimator & None & $E\left[h_{i} \mid \varepsilon_{i t}\right]$ \\
\hline Transient inefficiency estimator & $E\left[u_{i t} \mid \varepsilon_{i t}\right]$ & $E\left[u_{i t} \mid \varepsilon_{i t}\right]$ \\
\hline
\end{tabular}

Note: This table presents the distributional assumptions of the stochastic error and inefficiency components of the TREM and GTREM stochastic frontier models

(2014), Filippini and Greene (2016) propose to define a two-part disturbance term. Each part of the disturbance term is characterized by a skewed normal distribution with, in each case, one part assumed to be time-invariant and the other to be time-variant. The only difference between the TREM and GTREM setting therefore consists of the latter model containing a skewed normally instead of normally distributed time invariant disturbance term. ${ }^{19}$ The Filippini and Greene (2016) maximum simulated likelihood technique currently is preferred when $T$ is larger as it is in our empirical analysis (i.e., $T \geq 15$ ).

The firm's level of efficiency for the TREM is estimated using the conditional mean of the inefficiency term proposed by Jondrow et al. (1982). The firm's efficiency for the GTREM is estimated using the expression presented in Filippini and Greene (2016). Table 2 summarizes the econometric specification of the two models.

\footnotetext{
${ }^{19}$ It so far is not possible to consider determinants of inefficiency when estimating the GTREM proposed by Filippini and Greene (2016) as it would be possible with the TREM.
} 
Table 3 Cost function estimation results of the TREM and GREM specification

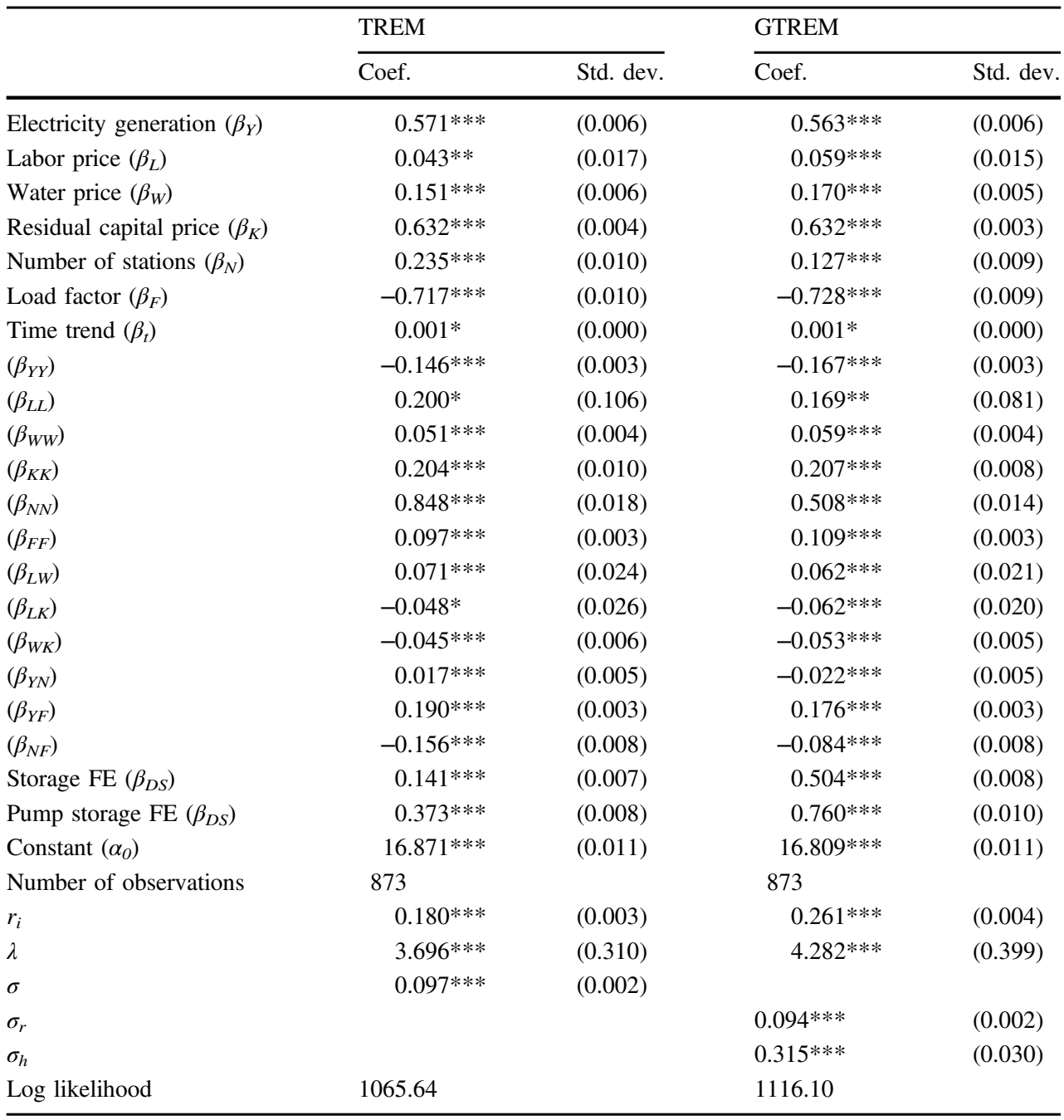

Note: This table presents the estimation results when applying the TREM and GTREM to the total cost function given in Eq. (2). FE abbreviates "fixed effect". The TREM and GTREM results are based on 200 draws each. Robust standard errors at the firm level are reported in parenthesis

Asterisks $* * *$ indicate significance at $1 \%$ level, $* *$ at $5 \%$ level and $*$ at $10 \%$ level

\section{Results}

\subsection{Cost function parameters}

The estimated coefficients of the two frontier models as well as their respective standard errors are listed in Table 3. Linear homogeneity was imposed a priori by normalizing prices and output with respect to the constant electricity price. To ensure monotonicity, microeconomic theory demands the cost function to be increasing in generated electricity and input prices. Furthermore, the function is expected to be concave with respect to input prices. Such concavity implies ownprice elasticities being negative with the Hessian matrix of second order partial derivatives of total costs with respect to prices being negative semi-definite. ${ }^{20}$ The cost function is

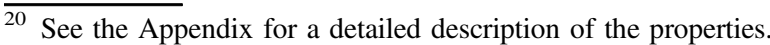

generally well behaved; except for the concavity condition (one of the four eigenvalue is greater than zero), our results obey these restrictions (cf. Tables 8 and 9 in the Appendix). We justify the slight violation of the concavity condition by the estimation of a behavioral cost function: the frontier cost model builds on the implicit assumption of firms not fully minimizing costs, which contradicts the concavity condition's underlying assumption of cost minimizing firms. ${ }^{21}$

The estimated coefficients in general have the expected sign and many are, together with lambda, ${ }^{22}$ statistically significant at a level of $1 \%$. The magnitude of the estimated coefficients is similar across both models. Technological progress in the hydropower sector is small; major

\footnotetext{
${ }^{21}$ See Bös (1989) for a discussion on behavioral cost functions.

${ }^{22}$ Lambda $(\lambda)$ expresses the ratio of the standard deviation of the inefficiency term $u_{i t}$ to the standard deviation of the stochastic term $v_{i t}$.
} 
technological components like turbines or dams can be considered as comparatively mature. Therefore, the low and partly insignificant coefficient estimate of the neutral, exogenous and progressive technical change $t$ is not surprising. ${ }^{23}$

The first order coefficients of the translog function are interpretable as elasticities at the sample median with the constant representing the total costs at the approximation point. The elasticity of the generated electricity is positive and highly statistically significant. The negative and statistically significant load factor indicates higher total costs for storage and pump storage firms compared to their run-ofriver counterparts, since the former technologies generally are characterized by comparatively low load factors. The firm-types fixed effects also point towards higher costs of storage and especially pump storage firms. Examples of factors contributing to these higher costs could be, next to the pump energy consumption of the latter type, relatively high investment costs for storage technologies in general, a higher complexity of operating such plants as well as their geographical remoteness.

\subsection{Cost efficiency}

Table 4 provides descriptive statistics of the estimated levels of cost efficiency. The median transient efficiency of the TREM of $94.9 \%$ is relatively similar in magnitude to the median transient result of the GTREM of $93.8 \%$. The dispersion of the estiamted transient efficiencies is slightly higher for the GTREM than for the TREM. As depicted by Fig. 2, mean efficiency estimates within the four quartiles of the yearly efficiency distributiatons are relatively constant across time, independently of the model specification. Hence, we find robust empirical evidence that Swiss hydro power firms on average neither strongly increased nor decreased their transient as well as persistent cost efficiency between 2000 and 2013.

The TREM and the persistent efficiency component of the GTREM measure different sorts of cost efficiency.

Table 4 Descriptive statistics of estimated cost efficiencies

\begin{tabular}{llll}
\hline & GTREM persistent & TREM & GTREM transient \\
\hline Mean & 0.862 & 0.936 & 0.920 \\
Min & 0.857 & 0.675 & 0.673 \\
Max & 0.888 & 0.994 & 0.991 \\
Std. dev. & 0.007 & 0.045 & 0.053 \\
25\% Pc. & 0.858 & 0.919 & 0.903 \\
Median & 0.859 & 0.949 & 0.938 \\
75\% Pc. & 0.863 & 0.966 & 0.955 \\
\hline
\end{tabular}

Note: This table presents descriptive statistics of the cost efficiency estimates of the TREM and GTREM frontier models. Statistics are based on the full sample of observations
Hence, the correlation between these two estimated efficiency levels is low and even negative (cf. Table 5). Accordingly, the correlation between the persistent and transient efficiency estimates of the GTREM is negative as well. In contrast, the correlation between the TREM cost efficiency and the transient efficiency of the GTREM is, as expected, positive and comparatively high. In conclusion, firms showing a high degree of persistent efficiency are not contemporaneously exhibiting production processes of a high degree of transient efficiency. The GTREM is our preferred model specification, because it allows for a simultaneous estimation of the level of persistent as well as transient cost efficiency. The predicted aggregate level of cost inefficiency of this model amounts to $21.8 \%(8.0 \%$ transient, $13.8 \%$ persistent) on average.

\subsection{Economies of density and scale}

The estimated coefficients reported in Table 3 can be used to compute the firms' level of economies of density and scale. Following the pioneering work of Caves et al. (1981, 1984), economies of density (ED) and economies of scale (ES) are estimated as

$$
\begin{gathered}
E D=\frac{1}{\partial \ln C / \partial \ln Y}, \\
E S=\frac{1}{\partial \ln C / \partial \ln Y+\partial \ln C / \partial N} .
\end{gathered}
$$

Economies of scale differ to economies of density (sometimes also called economies of spatial scale) in the assumption that an increase in firm size not only raises output, but to the same proportion also the number of plants under operation (Farsi et al. 2005). Economies of density and scale exist if the respective values of ED and ES are greater than 1 . Analogously, values smaller than 1 indicate diseconomies of density or scale.

Table 6 illustrates the descriptive statistics of the economies of scale and density computed for all firms in our sample and Table 7 presents the values for a small, medium and large hydropower firm. A small firm for instance is defined by values of $Y$ and $N$ that correspond to the first quartiles of the distribution of each variable. Accordingly, for the medium firm we use the median values of $Y$ and $N$ and for the large firm we use the respective third quartile values. The results reported in the two tables confirm the

\footnotetext{
${ }^{23}$ Filippini and Luchsinger (2007) find a significant effect of technical change in the Swiss hydropower sector of -0.018 . They estimate a translog variable cost model using seemingly unrelated regression and an unbalanced sample of 43 firms for the period of 1995 to 2002. In Banfi and Filippini (2010), statistically significant technical change amounts to -0.025 . They estimate a translog variable cost function applying a TREM specification and use the same data as Filippini and Luchsinger (2007).
} 

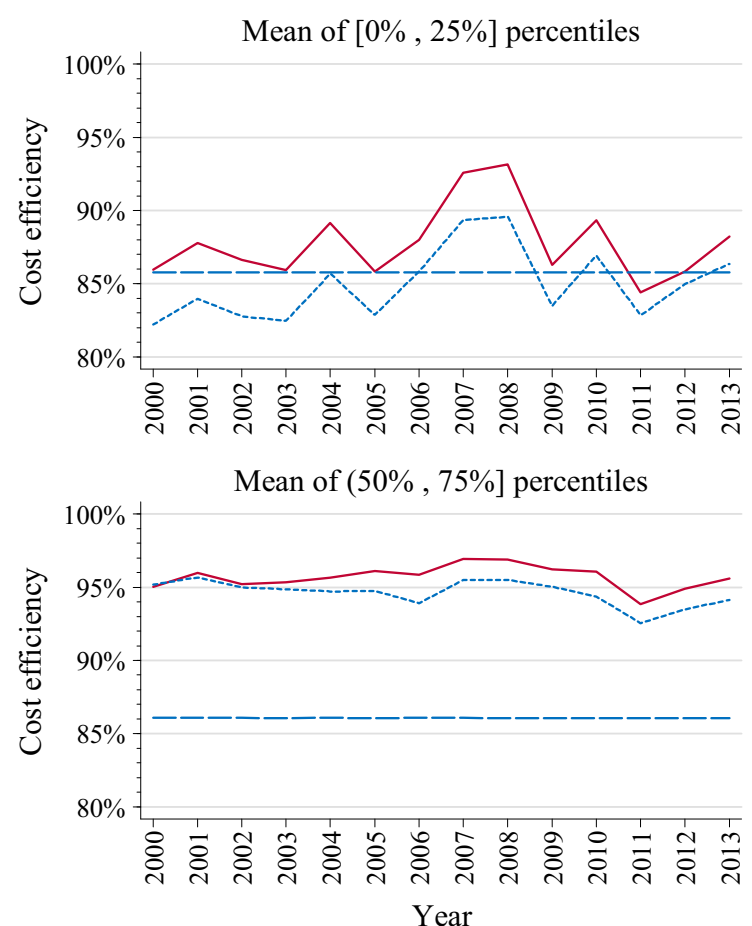

- TREM —— GTREM persistent ------- GTREM transient
Mean of $(25 \%, 50 \%]$ percentiles

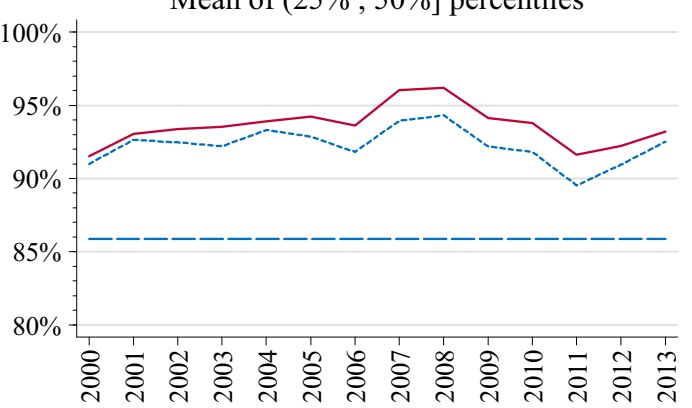

Mean of $(75 \%, 100 \%]$ percentiles

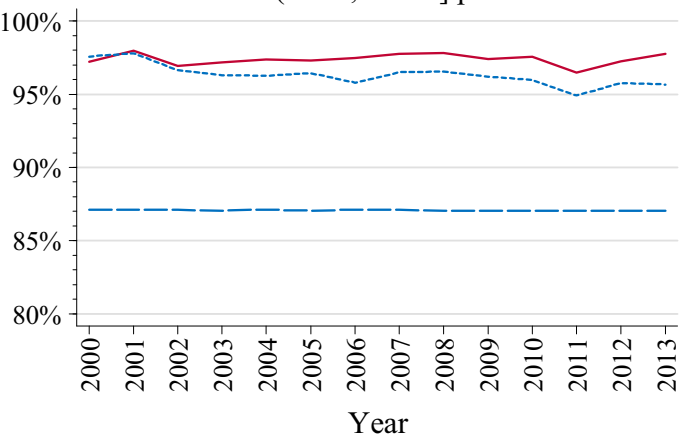

Fig. 2 Development of estimated cost efficiencies over time. Note: Fig. 2 presents the development of estimated cost efficiencies under the TREM and GTREM specification. For every individual year, firm

Table 5 Correlation coefficients of the efficiency estimates

\begin{tabular}{llll}
\hline & TREM & GTREM persistent & GTREM transient \\
\hline TREM & 1 & $-0.207\left[-0.110^{*}\right]$ & $0.819\left[0.748^{*}\right]$ \\
GTREM persistent & & 1 & $-0.694\left[-0.551^{*}\right]$ \\
\hline
\end{tabular}

Note: This table presents the correlation coefficients between estimated efficiencies of the TREM and GTREM frontier models. Spearman correlations are given in [.] brackets

Asterisks * indicate significance at a level of $5 \%$

Table 6 Economies of density (ED) and scale (ES) of the sample

\begin{tabular}{lcc}
\hline & TREM & GREM \\
\hline$E D$ & & \\
1st quartile & 1.286 & 1.417 \\
Median & 1.509 & 1.745 \\
3rd quartile & 1.944 & 2.250 \\
$E S$ & & \\
1st quartile & 1.127 & 1.083 \\
Median & 1.420 & 1.294 \\
3rd quartile & 1.794 & 1.917 \\
\hline
\end{tabular}

Note: This table presents the economies of density and scale when using estimates of the TREM and GTREM frontier models. Statistics are based on the respective first, second and third quartile firm observation level cost efficiency estimates are separated into quartiles. The figure shows the development of the yearly mean values of these quartiles

existence of positive economies of density and scale for most firms. $^{24}$

\section{Conclusions and discussion}

The goal of this paper was to estimate the persistent and transient cost efficiency levels in the Swiss hydropower sector applying two distinct frameworks: a true random effects model (TREM) and generalized true random effects model (GTREM). From a methodological point of view, the GTREM model seems to be interesting because it allows to simultaneously measure both types of efficiency, i.e., the persistent and transient one. The GTREM predicts the aggregate level of cost inefficiency to amount to $21.8 \%$ ( $8.0 \%$ transient, $13.8 \%$ persistent) on average between 2000 and 2013.

Results show that the Swiss hydropower sector is characterized by the presence of both, transient as well as persistent, cost inefficiencies. These inefficiencies are

\footnotetext{
$\overline{24}$ The study of Filippini and Luchsinger (2007) yields similar results. They estimate the economies of scale (but not economies of density) in the Swiss hydropower sector for the period 1995 to 2002 and find these scale economies to amount to 1.76 for small, 1.78 for medium and 1.76 for large firms.
} 
Table 7 Economies of density (ED) and scale (ES) of three typical firms

TREM GREM

$E D$

\begin{tabular}{lll} 
Small & 1.212 & 1.385 \\
Medium & 1.549 & 1.760 \\
Large & 2.004 & 2.292 \\
$E S$ & & \\
Small & 1.504 & 1.938 \\
Medium & 1.435 & 1.437 \\
Large & 1.518 & 1.327 \\
\hline
\end{tabular}

Note: This table presents the economies of density and scale when using estimates of the TREM and GTREM frontier models. Statistics are based on first, second and third quartile typical firms

different in absolute value and the negative correlations between them indicate that they indeed measure two kinds of inefficiencies, which differ in interpretation and implication. The transient component represents cost inefficiencies varying with time, e.g., inefficiencies stemming from a wrong adaption of production processes towards changing factor prices or singular management mistakes. On the other hand, the persistent part captures cost inefficiencies which do not vary with time, like inefficiencies due to recurring identical management mistakes, unfavorable boundary conditions for electricity generation or factor misallocations difficult to change over time.

The two types of cost efficiency allow a firm to elicit its cost saving potential in the short- as well as the long-run, but they might require a firm's management to respond with different improvement strategies. From a regulatory point of view, the results of this study could be used in the scope and determination of the amount of subsidies to be granted to a hydropower firm. Knowledge of the level of cost efficiency supports the government in avoiding a grant of subsidies to inefficient hydropower firms. For instance, the level of a subsidy could be reduced for hydro power firms showing a high level of cost inefficiency.

Acknowledgements We are grateful to the Bundesamt für Energie (BFE) for financially supporting a large study on the cost structure of the Swiss hydro power firms. The content of this paper does not necessarily represent the official views of BFE. All omissions and remaining errors are our responsibility. Furthermore, we would like to thank Nilkanth Kumar and the participants of the Mannheim Energy Conference 2015 and the 14th European Workshop on Efficiency and Productivity Analysis for their helpful comments.

\section{Compliance with ethical standards}

Conflict of interest The authors declare that they have no competing interests.

Open Access This article is distributed under the terms of the Creative Commons Attribution 4.0 International License (http://crea tivecommons.org/licenses/by/4.0/), which permits unrestricted use, distribution, and reproduction in any medium, provided you give appropriate credit to the original author(s) and the source, provide a link to the Creative Commons license, and indicate if changes were made.

\section{Appendix: Testing for monotonicity and quasi- concavity}

Linear homogeneity in factor prices of the cost function given in Eq. (2) implies

$c\left(Y, \lambda P_{L}, \lambda P_{W}, \lambda P_{K}, \lambda P_{E}\right)=\lambda c\left(Y, P_{L}, P_{W}, P_{K}, P_{E}\right) \mid \lambda>0$.

To reduce notation, unit $i$ and time $t$ subscripts are dropped. Homogeneity is imposed by dividing total costs and factor prices by the price of energy. Hence, what remains to be tested is the monotonicity and quasiconcavity of the cost function. Given the cost function of Eq. (2), the estimated cost share equations are

$$
\begin{gathered}
\frac{\partial \ln C}{\partial \ln P_{L}}=\hat{S}_{L}=\hat{\beta}_{L}+\hat{\beta}_{L L} \ln P_{L}+\hat{\beta}_{L W} \ln P_{W}+\hat{\beta}_{L K} \ln P_{K}, \\
\frac{\partial \ln C}{\partial \ln P_{W}}=\hat{S}_{W}=\hat{\beta}_{W}+\hat{\beta}_{W W} \ln P_{W}+\hat{\beta}_{L W} \ln P_{L}+\hat{\beta}_{W K} \ln P_{K}, \\
\frac{\partial \ln C}{\partial \ln P_{K}}=\hat{S}_{K}=\hat{\beta}_{K}+\hat{\beta}_{K K} \ln P_{K}+\hat{\beta}_{L K} \ln P_{L}+\hat{\beta}_{W K} \ln P_{W},
\end{gathered}
$$

Monotonicity is ensured if total costs are increasing in input prices as well as in output, i.e., if the following four conditions hold

$$
\begin{gathered}
\frac{\partial \ln C}{\partial \ln Y}=\hat{\beta}_{y}+\hat{\beta}_{Y Y} \ln Y+\hat{\beta}_{Y F} F+\hat{\beta}_{Y N} \ln N>0 \\
\text { and } \hat{S}_{L}>0 \text { and } \hat{S}_{W}>0 \text { and } \hat{S}_{K}>0 .
\end{gathered}
$$

Results of the evaluation of monotonicity at the sample's mean and median are shown in Table 8 . The results obey the restrictions noted in Eq. (3).

Concavity is given if the Hessian matrix of second order partial derivatives is negative semidefinite. According to Binswanger (1974, p. 380) the second order partial derivatives of a cost function can be derived as

$$
\frac{\partial^{2} C}{\partial P_{i} P_{j}}=\frac{C}{P_{i} P_{j}}\left(\beta_{i j}+S_{i} \cdot S_{j}\right)
$$

and $\frac{\partial^{2} C}{\partial P_{i}^{2}}=\frac{C}{P_{i}^{2}}\left(\beta_{i i}+S_{j}^{2}-S_{j}\right)$, where $i, j=\{L, W, K, E\}$. 
Table 8 Monotonicity at sample mean and median

\begin{tabular}{lcc}
\hline & TREM & GTREM \\
\hline $\begin{array}{lll}\text { Monotonicity at sample mean } \\
\hat{S}_{L}\end{array}$ & 0.057 & \\
$\hat{S}_{W}$ & 0.145 & 0.054 \\
$\hat{S}_{K} S$ & 0.631 & 0.166 \\
$\partial \ln C / \partial \ln Y$ & 0.873 & 0.638 \\
Monotonicity at sample median & & 0.783 \\
$\hat{S}_{L}$ & 0.064 & \\
$\hat{S}_{W}$ & 0.149 & 0.059 \\
$\hat{S}_{K}$ & 0.623 & 0.170 \\
$\partial \ln C / \partial \ln Y$ & 0.829 & 0.631 \\
\hline
\end{tabular}

Note: This table presents the estimated cost shares as well as the first derivative of total costs with respect to output of the TREM and GTREM frontier models evaluated at the sample mean or median

Hence, at the approximation point ${ }^{25}$ (the median), the Hessian matrix becomes

$\mathbf{G}=\left[\begin{array}{cccc}\hat{\beta}_{L L}+\hat{\beta}_{L}^{2}-\hat{\beta}_{L} & \hat{\beta}_{L W}+\hat{\beta}_{L} \cdot \hat{\beta}_{W} & \hat{\beta}_{L K}+\hat{\beta}_{L} \cdot \hat{\beta}_{K} & \hat{\delta}_{L E}+\hat{\beta}_{L} \cdot \hat{\delta}_{E} \\ \hat{\beta}_{L W}+\hat{\beta}_{W} \cdot \hat{\beta}_{L} & \hat{\beta}_{W W}+\hat{\beta}_{W}^{2}-\hat{\beta}_{W} & \hat{\beta}_{W K}+\hat{\beta}_{W} \cdot \hat{\beta}_{K} & \hat{\delta}_{W E}+\hat{\beta}_{W} \cdot \hat{\delta}_{E} \\ \hat{\beta}_{L K}+\hat{\beta}_{K} \cdot \hat{\beta}_{L} & \hat{\beta}_{W K}+\hat{\beta}_{K} \cdot \hat{\beta}_{W} & \hat{\beta}_{K K}+\hat{\beta}_{K}^{2}-\hat{\beta}_{K} & \hat{\delta}_{K E}+\hat{\beta}_{K} \cdot \hat{\delta}_{E} \\ \hat{\delta}_{L E}+\hat{\delta}_{E} \cdot \hat{\beta}_{L} & \hat{\delta}_{W E}+\hat{\delta}_{E} \cdot \hat{\beta}_{W} & \hat{\delta}_{K E}+\hat{\delta}_{E} \cdot \hat{\beta}_{K} & \hat{\delta}_{E E}+\hat{\delta}_{E}^{2}-\hat{\delta}_{E}\end{array}\right]$.

The $\delta$-coefficients are not estimated directly, due to the prior imposition of the homogeneity assumption. However, given the linear homogeneity constraints, they can be derived as

$$
\begin{gathered}
\hat{\delta}_{E}=1-\hat{\beta}_{L}-\hat{\beta}_{W}-\hat{\beta}_{K}, \\
\hat{\delta}_{L E}=0-\hat{\beta}_{L L}-\hat{\beta}_{L W}-\hat{\beta}_{L K}, \\
\hat{\delta}_{W E}=0-\hat{\beta}_{W W}-\hat{\beta}_{L W}-\hat{\beta}_{W K}, \\
\hat{\delta}_{K E}=0-\hat{\beta}_{K K}-\hat{\beta}_{L K}-\hat{\beta}_{W K}, \\
\hat{\delta}_{E E}=0-\hat{\delta}_{L E}-\hat{\delta}_{W E}-\hat{\delta}_{K E} .
\end{gathered}
$$

The vector of fitted factor shares is

$$
\mathbf{s}=\left[\begin{array}{c}
\hat{S}_{L} \\
\hat{S}_{W} \\
\hat{S}_{K} \\
\hat{S}_{E}
\end{array}\right],
$$

where $\hat{S}_{E}=1-\hat{S}_{L}-\hat{S}_{W}-\hat{S}_{K}$. The cost function is concave if the roots of the matrix $\mathbf{H}=\mathbf{G}+\mathbf{s} \cdot \mathbf{s}^{\prime}-\operatorname{diag}(\mathbf{s})$ are non-positive, e. if $\lambda_{i} \leq 0 \forall i=1, \ldots, 4$ with

\footnotetext{
$\overline{25}$ At the approximation point, all second order and interaction terms of a translog function collapse to zero.
}

Table 9 Roots of matrix $\mathbf{H}$ at sample mean and median

TREM GTREM

Concavity at sample mean

\begin{tabular}{lrr}
$\lambda_{1}$ & 0.325 & 0.240 \\
$\lambda_{2}$ & -0.000 & -0.000 \\
$\lambda_{3}$ & -0.220 & -0.156 \\
$\lambda_{4}$ & -0.365 & -0.414 \\
\multicolumn{2}{l}{ Concavity at sample median } & \\
$\lambda_{1}$ & 0.321 & 0.238 \\
$\lambda_{2}$ & 0.000 & -0.000 \\
$\lambda_{3}$ & -0.223 & -0.158 \\
$\lambda_{4}$ & -0.367 & -0.416 \\
\hline
\end{tabular}

Note: This table presents the roots of matrix $\mathbf{H}$ of the TREM and GTREM frontier models evaluated at the sample mean or median. Critical, i.e., positive values are given in italics

$\operatorname{det}\left(\mathbf{H}-\lambda \cdot \mathbf{I}_{4}\right)=0$. The roots of matrix $\mathbf{H}$ evaluated at the sample's mean and median are given in Table 9. In subsection 5.1 a justification is given for this slight violation of the concavity condition.

\section{References}

Aigner D, Lovell CAK, Schmidt P (1977) Formulation and estimation of stochastic frontier production function models. J Econom 6 (1):21-37

Badunenko O, Kumbhakar SC (2017) Economies of scale, technical change and persistent and time-varying cost efficiency in Indian banking: do ownership, regulation and heterogeneity matter? Eur J Oper Res 260(2):789-803

Banfi S, Filippini M (2010) Resource rent taxation and benchmarking - a new perspective for the Swiss hydropower sector. Energy Policy 38(5):2302-2308

Barros CP (2008) Efficiency analysis of hydroelectric generating plants: a case study for Portugal. Energy Econ 30(1):59-75

Barros CP, Peypoch N (2007) The determinants of cost efficiency of hydroelectric generating plants: a random frontier approach. Energy Policy 35(9):4463-4470

Barros CP, Chen Z, Managi S, Antunes OS (2013) Examining the cost efficiency of Chinese hydroelectric companies using a finite mixture model. Energy Econ 36(0):511-517

Battese GE, Corra GS (1977) Estimation of a production frontier model: with application to the pastoral zone of Eastern Australia. Aust J Agric Econ 21(3):169-179

Berndt ER, Christensen LR (1973) The translog function and the substitution of equipment, structures, and labor in US manufacturing 1929-68. J Econ 1(1):81-113

BFE (2013) Statistik der Wasserkraftanlagen der Schweiz (WASTA) 2000-2013. Bundesamt für Energie, Ittigen

BFS (2014) Entwicklung der Produzentenpreise, nach Wirtschaftszweigen und Art der Produkte, Indizes und Veränderungsraten zum Vormonat. Bundesamt für Statistik, Neuchâtel, http://www. bfs.admin.ch/bfs/portal/de/index/themen/05/04/blank/key/produzentenpreisindex.html. Accessed 11 Dec 2014 
Binswanger HP (1974) A cost function approach to the measurement of elasticities of factor demand and elasticities of substitution. Am J Agric Econ 56(2):377-386

Bös D (1989) Public enterprise economics: theory and application. North-Holland, Amsterdam

Butler JS, Moffitt R (1982) A computationally efficient quadrature procedure for the one-factor multinomial probit model. Econometrica 50(3):761-764

Caves DW, Christensen LR, Swanson JA (1981) Productivity growth, scale economies, and capacity utilization in U.S. railroads, 195574. Am Econ Rev 71(5):994-1002

Caves DW, Christensen LR, Tretheway MW (1984) Economies of density versus economies of scale: why trunk and local service airline costs differ. RJE 48:471-489

Chen Z, Barros CP, Borges MR (2015) A Bayesian stochastic frontier analysis of Chinese fossil-fuel electricity generation companies. Energy Econ 48:136-144

Christensen LR, Jorgenson DW, Lau LJ (1973) Transcendental logarithmic production frontiers. Rev Econ Stat 55(1):28-45

Coelli TJ, Rao DSP, O’Donnell CJ, Battese GE (2005) An introduction to efficiency and productivity analysis. Springer Science+Business Media, New York, NY

Colombi R, Kumbhakar SC, Martini G, Vittadini G (2014) Closedskew normality in stochastic frontiers with individual effects and long/short-run efficiency. J Prod Anal 42(2):123-136

Colombi R, Martini G and Vittadini G (2011) A stochastic frontier model with short-run and long-run inefficiency random effects. Working Papers Series No. 1101. Bergamo: Department of Economics and Technology Management, Universita di Bergamo

Cornwell C, Schmidt P, Sickles RC (1990) Production frontiers with cross-sectional and time-series variation in efficiency levels. $\mathrm{J}$ Econom 46(1-2): 185-200

Farsi M, Filippini M, Greene WH (2005) Efficiency measurement in network industries: application to the Swiss railway companies. J Regul Econ 28(1):69-90

Filippini M, Banfi S, Luchsinger C, Wild J (2001) Perspektiven für die Wasserkraft in der Schweiz-Langfristige Wettbewerbsfähigkeit und mögliche Verbesserungspotenziale, Studie im Auftrag des Bundesamtes für Energie, Bundesamtes für Wasser und Geologie und der Interessensgruppe Wasserkraft. Centre for Energy Policy and Economics, ETH Zürich, Zürich

Filippini M, Greene WH (2016) Persistent and transient productive inefficiency: a maximum simulated likelihood approach. J Prod Anal 45(2):187-196

Filippini M, Luchsinger C (2007) Economies of scale in the Swiss hydropower sector. Appl Econ Lett 14(15):1109-1113

Fried HO, Lovell CAK, Schmidt SS (2008) The measurement of productive efficiency and productivity growth. Oxford University Press, New York, NY
Friedlaender AF, Wang Chiang S-EJ (1983) Productivity growth in the regulated trucking industry. Res Transp Econ 1:149-184

Greene WH (2005a) Fixed and random effects in stochastic frontier models. J Prod Anal 23(1):7-32

Greene WH (2005b) Reconsidering heterogeneity in panel data estimators of the stochastic frontier model. J Econom 126 (2):269-303

Greene WH (2008) The econometric approach to efficiency analysis. In: Fried HO, Lovell CAK, Schmidt SS (ed.) The measurement of productive efficiency and productivity growth. Oxford University Press, New York, NY

Jondrow J, Knox Lovell CA, Ivan S. Materov, Peter S (1982) On the estimation of technical inefficiency in the stochastic frontier production function model. J Econom 19(2-3):233-238

Kumbhakar SC, Lien G, Hardaker JB (2014) Technical efficiency in competing panel data models: A study of Norwegian grain farming. J Prod Anal 41(2):321-337

Kumbhakar S, Lovell CAK (2000) Stochastic frontier analysis. Cambridge University Press, New York, NY

Liu CH, Lin SJ, Lewis C (2010) Evaluation of thermal power plant operational performance in Taiwan by data envelopment analysis. Energy Policy 38(2):1049-1058

Meeusen W, van Den Broeck J (1977) Efficiency estimation from Cobb-Douglas production functions with composed error. Int Econ Rev 18(2):435-444

Pitt MM, Lee L-F (1981) The measurement and sources of technical inefficiency in the Indonesian weaving industry. J Dev Econ 9 (1):43-64

See KF, Coelli T (2012) An analysis of factors that influence the technical efficiency of Malaysian thermal power plants. Energy Econ 34(3):677-685

Shrivastava N, Sharma S, Chauhan K (2012) Efficiency assessment and benchmarking of thermal power plants in India. Energy Policy 40:159-176

Sueyoshi T, Goto M, Ueno T (2010) Performance analysis of US coalfired power plants by measuring three DEA efficiencies. Energy Policy 38(4): 1675-1688

Tsionas EG, Kumbhakar SC (2014) Firm heterogeneity, persistent and transient technical inefficiency: a generalized true random-effects model. J Appl Econom 29(1):110-132

Yang H, Pollitt M (2009) Incorporating both undesirable outputs and uncontrollable variables into DEA: the performance of Chinese coal-fired power plants. Eur J Oper Res 197(3):1095-1105

Zellner A (1962) An efficient method of estimating seemingly unrelated regressions and tests for aggregation bias. J Am Stat Assoc 57(298):348-368 